\title{
Self-Assembly in Suspension
}

\section{H. Friedrich}

Laboratory of Materials and Interface Chemistry and Soft Matter CryoTEM Research Unit, Department of Chemical Engineering and Chemistry, Eindhoven University of Technology, P.O. Box 5135600 MB Eindhoven, The Netherlands, h.friedrich@tue.nl.

Over the past decade electron tomography (ET) has developed into an indispensable tool for the 3D characterization of nanostructured materials [1]. The recent interest, in particular by the physical and chemical sciences, is strongly connected to advances in shaping and patterning materials on the nanoscale. Synthesis of functional nanostructures is frequently carried out in solution/suspension and understanding and controlling the (self-) assembly process are key targets for academia and industry.

The ability of ET to give detailed insight into the 3D morphology of complex assemblies has considerably contributed to an understanding of their formation. For instance, ordered arrangements of nanoparticles, i.e. nanoparticle superlattices, can be formed from suspension by evaporation of the solvent. In addition to determining the local crystalline arrangement (Fig. 1), ET results indicated that superlattice nucleation and growth occurred at the suspension/air interface and that the unit cells of some lattices are anisotropically deformed upon drying [2]. For soft materials in particular, cryogenic preservation of nanostructures in their native, liquid environment is recommended as removal of the solvent leads to structural collapse [3]. An example of internally structured selfassembled nanospheres, i.e. cubosome, formed from a semicrystalline block copolymer, poly(ethylene oxide)-block-poly(octadecyl methacrylate) $\left(\mathrm{PEO}_{45}\right.$-b-PODMA 20 , in aqueous dispersion, is displayed in Fig. 2. Using cryo-ET it could be shown that internally bicontinuous $\mathrm{PEO}_{39}$-b-PODMA 17 aggregates undergo an unprecedented order-disorder transition of the microphase-separated domains that is accompanied by a change in the overall aggregate morphology [4]. Finally, the role of collagen in bone apatite formation was studied [5]. Combining cryo-EM with molecular modeling we show that collagen functions in synergy with inhibitors of hydroxyapatite nucleation to actively control mineralization (Fig. 3). In summary, (cryo)-electron tomography has become an indispensable tools for studying the (self-)assembly of materials in suspension [6].

References

[1] H. Friedrich, P. E. de Jongh, A. J. Verkleij, K. P. de Jong, Chem. Rev. 109 (2009) 1619.

[2] H. Friedrich, P.M. Frederik, G. de With, N.A.J.M. Sommerdijk Angew. Chem. Int. Ed. 49 (2010) 7850-7858.

[3] H. Friedrich, C.J. Gommes, K. Overgaag, J.D. Meeldijk, W.H. Evers, B. de Nijs, M.P. Boneschanscher, P.E. de Jongh, A.J. Verkleij, K.P. de Jong, A. van Blaaderen, D. Vanmaekelbergh Nano Lett. 9 (2009) 2719.

[4] B. E. McKenzie, F. Nudelman, P. H. H. Bomans, S. J. Holder, N. A. J. M. Sommerdijk J. Am. Chem. Soc. 132 (2010) 10256-10259.

[5] F. Nudelman, .K. Pieterse, A. George, P. H. H. Bomans, H. Friedrich, L. J. Brylka, P. A. J. Hilbers, G. de With, N. A. J. M. Sommerdijk Nat. Mater. 9 (2010) 1004-1009.

[6] HF acknowledges Prof. K. P. de Jong, Prof. D. Vanmaekelbergh, Dr. N. A. J. M. Sommerdijk, Dr. F. Nudelmann, Dr. S. J. Holder, Mr. P. H. H. Bomans, and Prof. G. de With for the fruitful 
collaborations. This research has been supported by the Netherlands Research School Combination Catalysis and Dutch Polymer Institute (\#688).

a

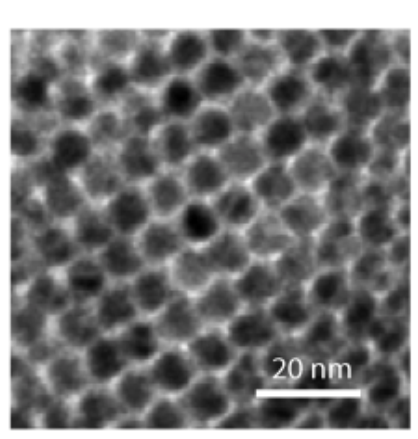

b

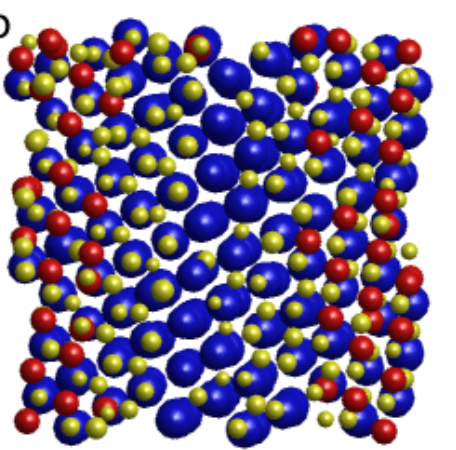

C

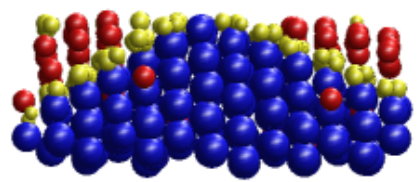

FIG. 1. Nanoparticle superlattice composed of PbSe and Au crystallites showing (a) TEM image of tilt series, (b) top view of segmented lattice, and (c) side view of segmented lattice. In the segmentation $\mathrm{PbSe}$ particles are shown in red and blue and Au particles in yellow.

a

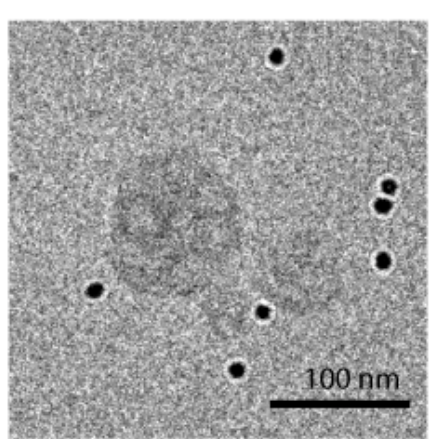

b

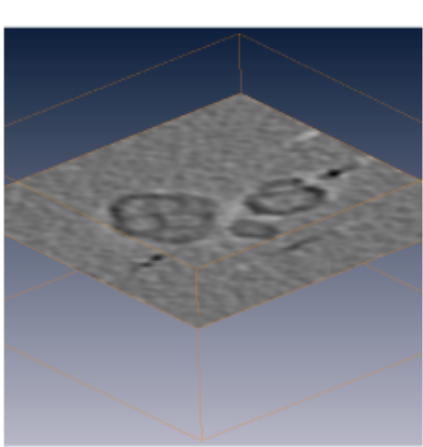

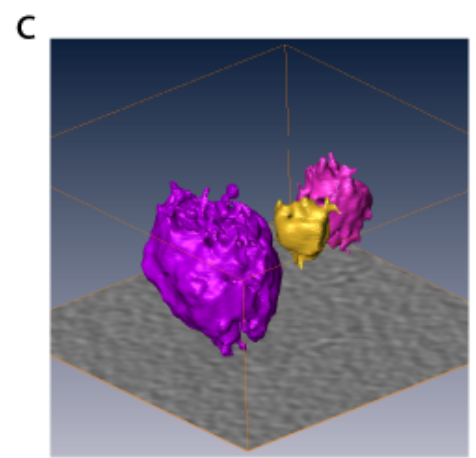

FIG. 2. Block copolymer aggregates $\left(\mathrm{PEO}_{45}-\mathrm{b}-\mathrm{PODMA}_{20}\right)$ showing (a) cryo-TEM image of tiltseries, (b) numerical cross section through reconstruction, and (c) isosurface rendering of aggregates.

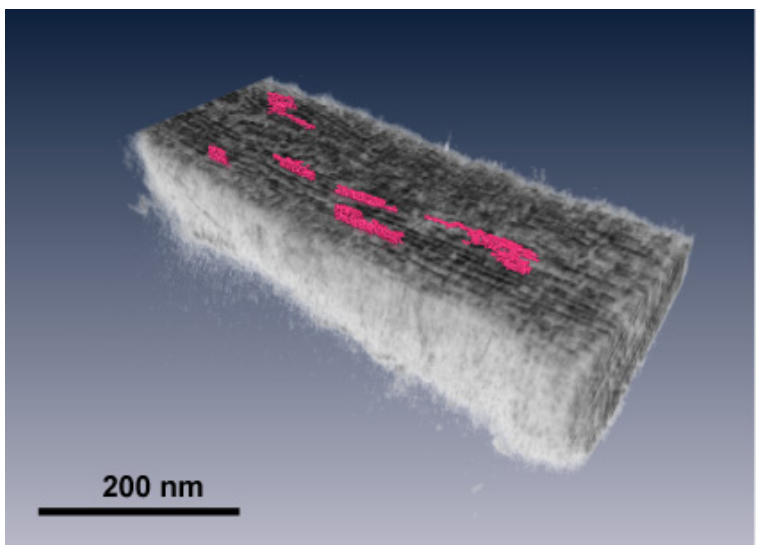

FIG. 3. Visualization of mineralized collagen revealing plate-shaped apatite crystals (pink) embedded in the collagen matrix. 